\title{
Resolución de un problema geométrico en torno a la noción de igualdad respecto a áreas
}

\author{
Geometric problem resolution around the notion \\ of equality with areas
}

Resolução de problemas geométricos em torno da noção de igualdade com as áreas

Recibido: mayo 2013

Aceptado: agosto 2013
Nelson Yampier Agudelo Peñuela ${ }^{2}$

Diego Fernando Escobar Salamanca ${ }^{3}$

\section{Resumen}

Se presenta experiencia de aula, sucedida -mediante la resolución de un problema geométrico- en el espacio de formación "problemas del álgebra y la geometría" de la carrera Licenciatura en Educación Básica con Énfasis en Matemáticas (LEBEM). Esta experiencia condujo a una noción de igualdad entre figuras geométricas, tomando como base los planteamientos de los elementos de Euclides. Siendo la resolución de problemas, la metodología mediante la cual se desarrolló la experiencia, metodología que permitió la construcción colectiva de un conocimiento, fruto de los desarrollos y puesta en juego de herramientas estrategias desarrolladas con base en los elementos, por parte de los estudiantes.

Palabras clave: Otras nociones de Educación Matemática; resolución de problemas; matemáticas escolares; geometría; geometría elemental; igualdad respecto a área.

\begin{abstract}
Classroom experience is presented, succeeded, by solving a geometric problem in space-training "problems of algebra and geometry" of the race Bachelor of Primary Education with Emphasis in Math (LEBEM). This experience led to a notion of equality of geometric figures, based on the ideas of Euclid's Elements. As the resolution of problems, the methodology by which developed the experience, methodology that allowed the collective construction of knowledge, the result of the development and implementation strategies developed toolkit based on the elements, by the students.
\end{abstract}

Keywords: Other notions of mathematics education, problem solving, school mathematics, geometry, elementary geometry, equal respect to area.

1 Artículo de Investigación.

2 Universidad Distrital Francisco José de Cáldas. Bogotá, Colombia. Contacto: nyampier.agudelo@gmail.com

3 Universidad Distrital Francisco José de Cáldas. Bogotá, Colombia. Contacto: dieguoescobar@gmail.com. 


\section{Resumo}

Experiência em sala de aula é apresentada, conseguiu, através da resolução de um problema geométrico de formação de espaço "problemas de álgebra e geometria" da Licenciatura corrida do Ensino Fundamental com Ênfase em Matemática (LEBEM). Esta experiência levou a uma noção de igualdade de figuras geométricas, com base nas idéias de Elementos de Euclides. Como a resolução de problemas, a metodologia pela qual desenvolveu a experiência, metodologia que permitiu a construção coletiva do conhecimento, o resultado das estratégias de desenvolvimento e implementação kit de ferramentas desenvolvidas com base nos elementos, por parte dos alunos.

Palavras-chave: Outras noções de educação matemática, resolução de problemas, a matemática da escola, geometria, geometria elementar, igual respeito à área.

\section{Contextualización}

La experiencia de aula que se relata a continuación se desarrolló en el marco del espacio de formación "Problemas del álgebra y la geometría" cursado por estudiantes para profesores, que se encontraban en el cuarto semestre del proyecto curricular LEBEM (de los cuales en ese entonces hacían parte quienes relatan la experiencia) de la Universidad Distrital Francisco José de Caldas. El espacio de formación en cuestión, al igual que la mayoría de los otros espacios de formación en el proyecto curricular, se desarrolló bajo un modelo de enseñanza constructivista enmarcado en la metodología de resolución de problemas. Luego de haber trabajado aproximadamente dos semanas concluyendo con la solución a un problema geométrico, el docente a cargo de espacio de formación planteó un nuevo problema también geométrico a los estudiantes. Dicho problema pedía la construcción de un polígono regular igual a otro dado, sin embargo ambas figuras deberían diferir en número de lados. Particularmente, dado un pentágono regular, construir un hexágono regular igual al pentágono. Fue entonces cuando los estudiantes pusieron sus esfuerzos en encontrar la solución empezando por entender qué noción de igualdad entre figuras planas permitiría que dos de ellas, iguales entre sí difirieran en número de lados.

\section{Referentes teórico-prácticos básicos}

Es pertinente mencionar que en los elementos de Euclides hay una concepción de igualdad entre polígonos (dada por la congruencia entre número y medida de lados y ángulos) implícitamente manejada a lo largo de las proposiciones, sin embargo "si las proposiciones I 43-45 nos introducen en el ámbito metódico de la trasformación de áreas, también abren la perspectiva de lo que se ha dado en llamar «álgebra geométrica» de los griegos (Vid. Supra. Introducción III, 2). El procedimiento permite asimismo añadir y sustraer áreas rectilíneas (...)". (Puertas, I99I) De esto se puede concluir que Euclides a pesar de no definir concretamente el concepto de igualdad (únicamente considerando el área), él lo maneja explícitamente porque transforma figuras, a las que cambia su forma logrando que conserven su área. Un ejemplo de lo anterior es la transformación que se puede realizar cuando se toma un triángulo y se construye un rectángulo con su misma área [I-42], por otra parte es posible construír un cuadrado con la misma área de un rectángulo [II-14].

Teniendo como base los elementos de Euclides, se pudo construir el pentagono regular, asimismo, el hexágono y cuadrados iguales a estas dos figuras. Lueg de esto, los intereses de los grupos respecto a la geometría elemental fueron:

- Razón y proporción. Definiciones [V-3] y [IV-12] respectivamente.

- Gnomón. Definición [II-2]

En esta experiencia estuvo presente un problema, por ende no se pretendía que a partir de la 
institucionalización del maestro se realizaran ejercicios de aplicación, ya que "Las aplicaciones y los problemas no se deben reservar para ser considerados solamente después de que haya ocurrido el aprendizaje, sino que ellas pueden y deben utilizarse como contexto dentro del cual tiene lugar el aprendizaje. El contexto tiene un papel preponderante en todas las fases del aprendizaje y la enseñanza de las matemáticas, es decir, no sólo en la fase de aplicación sino en la fase de exploración y en la de desarrollo, donde los alumnos descubren o inventan las matemáticas". (MEN,1998), a lo largo de este experiencia es evidente que los estudiantes resolutores del problema hacen un gran esfuerzo por entender las definiciones de los diferentes conceptos matemáticos en juego además dan una solución al problema pensando en estas definiciones aplicadas a las necesidades del problema, por lo tanto se puede inferir que el problema planteado en el curso es un problema pensado no para la aplicación de un algoritmo sino en la construcción de un conocimiento a través de la exploración y el desarrollo de la situación dentro de un contexto académico donde la pretensión es que a partir de una interpretación geométrica de la situación se encuentre una estructura relacionada con las características algebraicas de las figuras.

Para Guzmán la resolución de un problema pasa por cuatro fases:

1. La familiarización con el problema.

2. La búsqueda de estrategias.

3. El desarrollo de la estrategia.

4. La revisión del proceso." (Blanco,1996).

$\mathrm{Al}$ ser así, los resolutores de este problema se familiarizan con el éste al entender las definiciones de algunos conceptos desde los elementos de Euclides, en base a estas definiciones buscan la trasformación de las figuras que conservan su área, lo cual permite llegar a la solución del problema, y se remiten a las proposiciones para realizar la demostración y rectificar los resultados obtenidos.

\section{Descripción general de la experiencia}

Una vez planteado el problema, los estudiantes organizados en grupos de 3 o 4 integrantes procedieron a realizar esfuerzos en pro de la resolución del problema.

En primer tópico generalizado en los diversos grupos de estudiantes, fue la noción de igualdad que debería ser usada en el problema, ya que si bien, ambas figuras deberían ser iguales una debía ser un pentágono mientras la otra un hexágono y, la noción de igualdad entre polígonos intuitiva en los estudiantes, correspondía a la congruencia dada por el número de lados, la medida de éstos y la medida de los ángulos. Finalmente se llegó a la conclusión de que la igualdad, en el caso del problema, solamente debería contemplar la cantidad de magnitud área de ambos polígonos, debido a que Euclides en una de sus proposiciones afirma hacer un cuadrado igual a un rectángulo, proposición cuya demostración permite deducir que la igualdad se refiere únicamente a la superficie. Luego de que varios grupos llegaron a esta conclusión, el docente encargado realizó una institucionalización sobre éstos avances.

Posteriormente algunos grupos llegaron a entender procesos euclídeos para convertir cualquier figura plana en un rectángulo. Estos grupos realizaron una socialización de sus avances, luego de lo cual el problema para todo el curso se redujo a encontrar una forma de hacer un rectángulo igual a un cuadrado de área dada, pero dicho rectángulo debía ser semejante a un rectángulo específico construido a partir de la razón entre el lado y la apotema de un hexágono.

Después de la simplificación del problema, uno de los grupos (al que pertenecía uno de los estudiantes que relatan la experiencia) desarrolló con fundamentos de la geometría elemental, un método para construir con el área de un cuadrado dado, un rectángulo semejante a uno dado. Haciendo primero un cuadrado igual al rectángulo dado, y sobre esta 
construcción montar una semejante teniendo como parámetro al cuadrado dado. Luego de lo cual dicho grupo socializó el método mencionado.

Finalmente la socialización del grupo al que se hizo referencia anteriormente, sirvió a los demás grupos para dar solución al problema, luego de ésto cada grupo socializó con el resto del curso sus caminos particulares de resolución del problema ayudados finalmente por el método para construir con el área de un cuadrado dado, un rectángulo semejante a uno dado.

\section{Logros y dificultades evidenciadas}

Dentro de los logros conseguidos en la experiencia, se puede mencionar el hecho de que la revisión del proceso y la comunicación de los avances respecto al desarrollo del problema, constituyeron un ambiente de discusión y validación de los mismos, siendo los demás estudiantes individuos colaboradores, aun cuando no pertenecían a los grupos que comunicaban sus ideas. También dichos avances potenciaron el proceso de otros grupos que por entonces se encontraban atascados en cierto punto de la resolución del problema, pero al conocer de las estrategias utilizadas por otros grupos, pudieron avanzar y ser capaces posteriormente de comunicar a sus pares alguna estrategia que les fuese útil. Como dificultad se puede resaltar el hecho de que los estudiantes no tienen como hábito un trabajo en grupo en el cual cada integrante realice aportes y proponga estrategias de solución, muchas veces resultó ser solo uno de los miembros de un grupo, el que en realidad se preocupó e interesó en la resolución del problema.

\section{Reflexión final}

La geometría elemental, un estudio de las magnitudes continuas, fue construida por Euclides de tal manera que partiendo de algo tan básico como los segmentos, y del triángulo como operador aditivo, crea los ángulos y posteriormente las figuras planas, además establece métodos para sumar y restar segmentos y ángulos, luego mediante la proposición [I-47] establece un método para sumar y restar figuras planas de manera homogénea, es decir, suma o resta cuadrados y obtiene como resultado cuadrados. Pero, ¿bajo qué criterios es capaz de sumar figuras planas? Y ¿qué sucede si los polígonos que se quieren sumar no corresponden a cuadrados? El proceso realizado por el curso de "problemas del álgebra y a geometría" en la resolución del problema del hexágono igual un pentágono, puede evidenciar la posibilidad de realizar suma de figuras planas no cuadradas mediante la transformación de éstas en cuadrados y viceversa bajo el criterio de igualdad de polígonos respecto a sus áreas, trabajado por Euclides. También puede llenar de sentido y conocimiento formal a los futuros docentes de matemáticas, la posibilidad de realizar mediciones de superficies con unidades no cuadradas, además, queda como experiencia que el método de resolución de problemas es capaz de generar en un ambiente propicio, la construcción colectiva de un conocimiento.

\section{Referencias}

Blanco, J. (1996, febrero). La resolución de problemas. Una revisión teórica. SUMA. Recuperado de http://revistasuma.es/IMG/ pdf/21/011-020.pdf

MEN. (1998). Serie lineamientos curriculares. Bogotá

Puertas, M. (Ed.). (1991). Elementos Libros I-IV. Madrid: Gredos. 